\title{
Uygur Sivil Belgeleri Üzerine Çince Çalışmalar
}

\author{
Hatice Veli*
}

Özet: Literatürde "Uygur sivil belgeleri”, "din dışı belgeler", "sözleșme belgeleri", "hukuk belgeleri" gibi farklı adlarla adlandırılan belgelerin çoğu Koço Uygur Hanlı̆ğ dönemine ve Uygurların Moğolların egemenliği altına girdiği dönemlere ait belgelerdir. Her ne kadar yazıldığı tarih belirtilmese de bu belgelerin 9.- 14. yüzyıllara uzanacak kadar geniş bir zaman dilimi içerisinde kaleme alındığ ${ }^{\prime}$ düşünülmektedir. $\mathrm{Bu}$ belgeler İslamiyet'ten önceki Uygurların siyasi, iktisadi ve kültürel yaşamıyla ilgili önemli bilgiler barındırdığından dolayı dil, tarih ve kültür araştırmaları için son derece kıymetli malzemelerdir. Söz konusu belgeler üzerinde Japonya ve Almanya bașta olmak üzere birçok ülkede oldukça titiz çalışmalar yürütülmüştür. Uygur sivil belgeleri üzerine Çin'de yapılan çalışmalar da azımsanmayacak kadar önemli sayıdadır. Bu makalede, Uygur sivil belgeleri üzerine Çin'deki çeşitli bilimsel yayınlarda neşredilmiş çalışmalardan Çince olanlar kronolojik sırayla ele alınmaya çalışıııışıır.

Anahtar Sözcükler: Uygur Sivil Belgeleri, Koço Uygur Hanlığı, Uygurlar, Çin'deki Uygur Çalışmaları, Çince Uygur Çalışmaları

\begin{abstract}
A Bibliography of Works in Chinese on Uyghur Civil Documents

Most of the documents called "Uyghur civil documents", "nonreligious documents", "contract documents" and "legal documents" in the literature belong to the Kocho Uyghur Khanate period and the periods when the Uyghurs came under the rule of the Mongols. Even though the date of writing is not specified, it is thought that these documents were written in a wide period of time between $9^{\text {th }}$
\end{abstract}

*Dr., haticeveli78@gmail.com; ORCID: 0000-0002-1207-9217.

Received: 03.11.2021; $\quad$ Accepted: 26.12.2021;

Published: 31.12 .2021 
and $14^{\text {th }}$ centuries. Since these documents contain important information about the political, economic and cultural life of Uyghurs before Islam, they are very valuable materials for language, history and culture studies. Meticulous study has been carried out on these documents in many countries, especially in Japan and Germany. Publications on Uyghur civil documents in China are also significant. In this paper, academic works on Uyghur civil documents published in various scientific publications in China in Chinese are cited in chronological order.

Key Words: Uyghur Civil Documents, Koço Uyghur Khanate, Uyghurs, Uyghur Studies in China, Uyghur Studies in Chinese.

Giriş: Budist, Manihaist ve Hristiyan içerikli eserlerden farklı olarak Uygurların sosyal, iktisadi ve kültürel yaşamıyla ilgili konular işlendiğinden dolayı, literatürde "Uygur din dışı belgeleri", "sivil belgeler", "sözleşme belgeleri" ve "hukuk belgeleri” gibi farklı isimlerle adlandırılan belgelerin tarihlendirilmesi muhtemelen 9.- 14 . yüzyıllara dayanmaktadır.

Belgeler Doğu Türkistan'daki Tarım havzasında bulunmuştur. Bunların büyük çoğunluğu İdikut (Koço) şehri kalıntıları etrafındaki kazılarda ve Ürümçi çevresinde ortaya çıkmıştır. Ayrıca Turfan vahasındaki Astana, Bezeklik, Bulayık, Çigtim, Murtuk, Sengim, Toyok, Yarhoto'da ele geçenler de bulunmaktadır. Bununla birlikte pek çok belgenin hangi bölgeden geldiği ve kaynağı tam olarak bilinmemektedir (Özyetgin 2015: 20)

Geçen yüzyılın başında Rus, İngiliz, Alman, Japon, Fransız gibi birçok araştırmacının Doğu Türkistan'a düzenlediği seferleri esnasinda bulunan belgeler; Berlin ${ }^{1}$, Leningrad, Kyoto, Londra, Paris, Helsinki, Pekin, Ürümçi ve İstanbul'daki çeşitli kütüphanelerde muhafaza edilmektedir (Arat 1964: 13-18).

Uygur sivil belgeleri muhteva açısından çeşitlilik göstermektedir. Belgeler üzerinde ilk tasnif çalışması yapan Reşit Rahmeti Arat'tır (1964). Daha sonra İzgi (1987: 56) ve Clark'1n

1 Almanya'daki Uygur sivil belgelerinin kataloğu için bkz. Simone-Christiane Raschmann Alttürkische Handschriften, Teil 13: Dokumente, Teil 1. (VOHD, XIII-21), Stuttgart, Franz (2007). 
(1975) da tasnif çalışmaları bulunmaktadır. Bunun yanında Li Jingwei (1996), Geng Shimin (2006), Yüsüp ve Sayit (2000) de çalışmalarında ele aldıkları sivil belgeler üzerine farklı tasnifler yapmışlardır. Uygur sivil belgeleri üzerine yapılan en önemli çalışmalardan biri Nobuo Yamada'nın ölümünden sonra Juten Oda, Peter Zieme, Hiroshi Umemura ve Takao Moriyasu tarafindan yayıma hazırlanan Sammlung Uigurischer Kontrakte I-III adlı eserdir. Bu eserde toplam 121 belge ele alınmış olup belgeler satış belgeleri, değiş-tokuş belgeleri, kiralama, borç alıp-verme, evlat edinme, güvence, serbest birakma, vasiyet ve diğer belgeler olmak üzere farklı dokuz gruba ayrılmıştır.

Uygur sivil belgeleri içerikleri bakımından; bireylerin kendi aralarında yaptıkları anlaşmalar ve bireylerin devletle olan ilişkilerini ortaya koyan belgeler olarak ikiye ayrılır. Bireylerin kendi aralarındaki ilişkilerle ilgili olanlar, vasiyetnameler, köle satışları, çocuğun evlatlık verilmesi, arazi satışları, arazi kiraya verme gibi konuları içermektedir. Bireylerin devletle olan ilişkileri için düzenlenen vesikalar ise nüfus sayımı ve vergilerle ilgilidir (İzgi 2014: 302).

Uygur sivil belgeleri üzerine yapılan ilk önemli bilimsel çalışma 1928 yılında yayımlanan W. Radloff'a ait Uigurische Sprachdenkmäler adlı çalışmadır. 128 Uygur yazılı belgenin bulunduğu bu eser W. Radloff'un ölümünden sonra öğrencisi S.E. Malov tarafından yayımlanmıştır. Eserde her belgenin dökme Uygur harfleriyle metinleri verilmiştir. Birçok hatalı okuma bulunmasına rağmen Radloff'un bu çalışması Uygur sivil belgeleri alanında yapılan ilk çalışma olması açısından önem arzetmektedir. Uygur sivil belgeleri üzerine Rusça neşredilmiş yayınlar arasında yakın zamanlarda yapılan önemli bir metin çalışması L.Yu. Tuguşeva'nın Uygurskie Delovie Dokumentı, X-XIV vv. $\dot{I} z$ Vostoçnogo Türkistana, Moskova, (2013) adlı çalışmadır.

Uygur sivil belgeleri kültür ve tarih çalışmaları yanında, başta Eski Uygurca olmak üzere dil çalışmaları açısından da büyük önem taşımaktadır. Belgeler Uygurların günlük hayatlarının farklı yönleriyle ilgili sözcükler ve terimler içerdiği için Eski Uygurca 
sözvarlığı ve karşılaştırmalı sözvarlığı çalışmaları için çok değerli kaynaklardir ${ }^{2}$.

Uygur sivil belgeleri üzerine Çin'de de oldukça titiz ve önemli çalışmalar yapılmasına rağmen bunlardan birkaç tanesi dışındakiler pek bilinmemektedir. Makalemizde Uygur sivil belgeleri üzerine Çin'de yapılan çalışmalardan Çince olanlara kronolojik olarak yer verilmiştir.

\section{Uygur Sivil Belgeleri Üzerine Çince Çalışmalar (1954-2021)}

\section{4}

冯家升 Feng Jiasheng, “元代畏兀儿文契约二种” (Yuandai weiwuerwen qiyue er zhong) [Yuan Dönemine Ait İki Uygurca Anlaşma], Lishi yanjiu, 1954, sayı 1, s. 119-131.

\section{8}

冯家升 Feng Jiasheng, “回鹘文斌通（善斌)” (Huihuwen Bintong (Shanbin)) [Uygurca Bintung Vesikas1]. Kaogu xuebao, 1958, s. 109-120.

冯家升 Feng Jiasheng, F. Tenişev. “回鹘文斌通（善斌）卖身契 三种 - 附控告主人书” (Huihuwen Bintong (Shanbin) maishen qi san zhong - fu konggao zhuren shu) [Bintung'un Satılmasına Dair Uygurca Vesikasının Üçüncüsü-Sahibini Şikâyet Etme Belgesi], Kaogu xuebao, 1958, say1 2, s. 109-120.

\section{0}

冯家升 Feng Jiasheng，“回鹘文契约二种” (Huihuwen qiyue er zhong) [İki Uygurca Anlaşma], Wenwu, 1960, sayı 6, s. 32-34.

2 Belgelerin sözvarlığının Türkiye Türkçesi, Türkiye Türkçesi ağızları, Yeni Uygurca, Özbekçe, Sarı Uygurca, Tuvaca, Hakasça, Altayca ve Yakutçadaki eş anlamlı veya eş sesli biçimleriyle karşılaştırmaları için bkz. Ayazlı 2016. 


\section{8}

耿世民 Geng Shimin, “两件回鹘文契约的考释” (Liang jian huihuwen qiyue de kaoshi) [İki Uygurca Anlaşma Üzerinde Açılama], Zhongyang minzu xueyuan xuebao, 1978, sayı 2, s. 43 49.

耿世民 Geng Shimin, “回鹘文摩尼教寺院文书初释” (Huihuwen monijiao siyuan wenshu chu shi) [Uygurca Mani Dini Tapınak Belgesi Üzerinde İlk Açılama], Kaogu xuebao, 1978, sayı 4, s. 497-516.

\section{0}

耿世民 Geng Shimin, “几件回鹘文文书的译释” (Jijian huihuwen wenshu de yishi) [Birkaç Uygurca Vesikanın Tercümesi ve Açıklaması], Wenwu, 1980, sayı 5, s. 83-84.

Kurban Veli，“吐鲁番出土的五件回鹘文文书” (Tulufan chutu de wujian huihuwen wenshu) [Turfan'da Bulunmuş Beş Uygurca Belge], Zhongguo mizu guwenzi yanjiu, 1980, s. 105-113.

\section{1}

耿世民 Geng Shimin, “两件回鹘文买卖奴隶文书的考释” (Liangjian huihuwen maimai nuli wenshu de kaoshi) [Köle AlımSatımına Dair İki Uygurca Vesika Üzerinde Açıklama], Minzu Yuwen Lunji, Zhongguo shehui kexue chubanshe, 1981, s. 272-291.

\section{3}

张承志 Zhang Chenzhi, “元代畏兀儿人内部状况” (Yuandai weiwu'erren neibu zhuangkuang) [Yuan Dönemindeki Uygurların İç Durumu], Minzu yanjiu, 1983, sayı 5, s. 13-23. 


\section{7}

袁丁 Yuan Ding, “回鹘文社会经济文书选注（一)” (Huihuwen shehui jingji wenshu xuanzhu-(yi)) [Uygurca Sosyo-Ekonomik Vesikalardan Seçmeler-1], Kashi shifan xueyuan xuebao, 1987, say1 2, s. 64-79.

\section{8}

杨富学 Yang Fuxue, “元代畏兀儿税役考略” (Yuandai weiwu'er shuiyi kaolue) [Yuan Dönemindeki Uygurların Vergi ve Angarya Yükümlülüğü Üzerine], Tujue xibei minzu yanjiu, 1988, sayı 2, s. 121-126.

张铁山 Zhang Tieshan “苏联所藏编号 SJ Kr·4 /638 回鹘文文书 译释”, (Sulian suo cang bianhao SJ Kr·4 /638 huihuwen wenshu yishi) [Sovyetler Birliğinde SJ Kr·4 /638 Koduyla Muhafaza Edilen Uygurca Belgelerin Tercümesi ve Açıllaması Üzerine], Xinjiang daxue xuebao, 1988, sayı 4, s. 96-106.

张铁山 Zhang Tieshan, “我国回鹘文及其文献研究概述” (Woguo huihuwen jiqi wenxian yanjiu gaishu) [Ülkemizde (Çin'de) Uygurcanın ve Uygurca Vesikaların Araştırılma Durumu Üzerine], Kashi shifan xueyuan xuebao, 1988, sayı 2, s. 65-83.

孙振玉 Sun Zhenyu, “从古文书看高昌摩尼教——对“回鹘文 摩尼教寺院文书, 再研究” (Cong gu wenshu kan gaochang monijiao —Dui 'huihuwen monijiao siyuan wenshu' zai yanjiu) [Kadim Eserlerden Turfan'daki Mani Dinine Bakış 'Uygurca Mani Dini Tapınak Belgesi' Üzerinde Yeniden Araştırma], Xibei shidi, 1988, sayı 3, s. 21- 28. 
杨富学 Yang Fuxue, “元代回鹘文献——农奴免赋请愿书研究” (Yuandai huihu wenxian- nongnu mianfu qingyuanshu yanjiu) [Yuan Dönemindeki Uygurca Vesikalar-Çiftçi Kölelerin Vergi Muafiyeti Talebi ile İlgili Belge Üzerinde Araştırma], Xinjiang wenwu, 1988, say1 4, s. 69-77.

\section{9}

牛汝极 Niu Ruji, “四件敦煌回鹘文书信文书” (Sijian dunhuang huihuwen shuxin) [Dunhuang'da Bulunmuş Dört Uygurca Mektup Üzerine]. Dunhuang yanjiu, 1989, say1 1, s. 104-108.

牛汝极 Niu Ruji , “四封 9-10 世纪的回鹘文书信译考” (Sifeng 9-10 shiji de huihuwen shuxin yikao) [9-10.Yüzyllara Ait Dört Uygurca Mektubun Tercümesi ve Tahlili], Xinjiang daxue xuebao, 1989, say1 2, s. $96-100$.

杨富学 Yang Fuxue, “古代新疆实物货币———粗棉布” (Gudai xinjiang shiwu huobi-cu mianbu) [(Kadim Xinjiang'daki Nakdi Para- Kaba Böz], Zhongguo qianbi, 1989, sayı 3, s. 15-17. 杨富学 Yang Fuxue, “回鹘文文书中所见元代畏元儿租佃契约关 系研究” (Huihuwen wenshu zhong suojian yuandai weiwu'er zudian qiyue guanxi yanjiu) [Uygurca Belgeler Temelinde Yuan Dönemindeki Uygurların Kiralama İlişkileri Üzerine Araştırma], Xibei minzu yanjiu, 1989, say1 2, s. 161-172.

张铁山 Zhang Tieshan, “我国收藏刊布的回鹘文文献及其研究” (Woguo shoucang kanbu de huihuwen wenxian jiqi yanjiu) [Ülkemizde (Çin'de) Muhafaza Edilen ve Yayımlanan Uygurca Belgeler Üzerine], Xinjiang shehui kexue, 1989, sayı 4, s. 98-105. 
袁丁Yuan Ding, “回鹘文社会经济文书选注(四) 一 有关赋税、 徭役的文书” (Huihuwen shehui jingji wenshu xuanzhu (si) youguan fushui, yaoyi de wenshu) [Uygurca Sosyo-Ekonomik Vesikalardan Seçmeler 4 - Vergi ve Angarya ile İlgili Belgeler], Kashi shifan xueyuan xuebao, 1989, say1 1, s. 52-67.

\section{0}

牛汝极 Niu Ruji, 杨富学 Yang Fuxue ,“从一份摩尼文文献谈高 昌回鹘的几个问题” (Cong yifen moniwen wenxian tan gaochang huihu de jige wenti) [Mani Yazısıyla Yazılmış Bir Belge Temelinde Koço Uygurları İle İlgili Birkaç Meseleye Bakış], Kashi shifan xueyuan xuebao, 1990, say1 4, s. 46-53.

杨富学 Yang Fuxue, “吐鲁番出土回鹘文借贷文书概述” (Tulufan chutu huihuwen jiedai wenshu gaishu) [Turfan'da Bulunmuş Uygurca Ödünç Alma Vesikaları Üzerine], Dunhuang yanjiu, 1990, sayı 1, s. 77-84.

杨富学 Yang Fuxue, “蒙元时代高昌回鹘土地制度初探” (Mengyuan shidai gaochang huihu tudi zhidu chutan) [Moğol-Yuan Sülalesi Döneminde Koço Uygurlarının Toprakla İlgili Uygulamaları Üzerinde İlk Araştırmalar], Tulufanxue yanjiu, zhuanji, 1990, s. 279-318.

杨富学 Yang Fuxue, “两件回鹘文敕令译释” (Liangjian huihuwen chiling yishi) [İki Uygurca Fermanın Tercümesi ve Açıklamas1], Xinjiang wenwu, 1989, sayı 4, s. 87-93.

郭平梁 Guo Pingliang, “高昌回鹘社会经济管窥” (Gaochang huihu shehui jingji guankui) [Turfan Uygurlarının SosyoEkonomisine Bakış], Xinjiang shehui kexue, 1990, sayı 2, s. 82-95. 
袁丁 Yuan Ding, “回鹘文社会经济文书选注（一）, 续补”, (Huihuwen shehui jingji wenshu xuanzhu (yi) 'xubu') [Uygurca Soyo- Ekonomik Vesikalardan Seçmeler 1 - İlave], Kashi shifan xиеуиап хиеbao, 1990, say1 2, s. 51-67.

\section{1}

Takao Moriyasu. Çev: Yang Fuxue, Huang Jianhua, “敦煌出土元 代回鹘文佛教徒书简” (Dunhuang chutu yuandai huihuwen fojiaotu shujian) [Dunhuang'da Bulunmuş Yuan Sülalesi Dönemine Ait Uygur Budist Rahiplerinin Mektuplar1], Dunhuang уапјіu, 1991, say1 2, s. 37-48.

李经纬 Li Jingwei, “回鹘文借贷文书选注” (Huihuwen jiedai wenshu xuanzhu) [Uygurca Borç Alma Vesikalarından Seçmeler], Xibei minzu yanjiu, 1991, say1 2, s. 31-59.

李经纬 Li Jingwei, “回鹘文文献语言的数量词” (Huihuwen wenxian yuyan de shuliangci) [Uygurca Vesikalarda Sayı ve Ölçü Birimleri], Yuyan yanjiu, 1991, sayı 1, s. 8-12.

\section{2}

牛汝极 Niu Ruji, “六件 9 〜 10 世纪敦煌回鹘文商务书信研究” (Liujian $9 \sim 10$ shiji dunhuang huihuwen shangwu shuxin yanjiu) [9. 10. Yüzyıllara Ait, Dunhuang'da Bulunmuş Uygurca Altı Ticaret Mektubu Üzerinde Araştırma], Xibei minzu yanjiu, 1992, sayı 1, s. 67-79.

牛汝极 Niu Ruji, 杨富学 Yang Fuxue, “回鹘文摩尼教寺院文书 释文的几处商榷” (Huihuwen monijiao siyuan wenshu shiwen de jichu shangque) [Uygurca Mani Dini Tapınak Belgesinin 
Tercümesiyle İlgili Birkaç Görüş], Xiyu shidi, 1992, sayı 4, s. 4046.

杨富学 Yang Fuxue，“回鹘文书所见高昌回鹘王国的纸钞与铸币” (Huihu wenshu suojian gaochang huihu wangguo de zhichao yu zhubi) [Uygurca Vesikalarda Adı Geçen Koço Uygur Devletindeki Kâğıt Paralar ve Maden Paralar], Zhongguo shehui jingjishi yanjiu, 1992 yıl, sayı 1, s. 8-14.

杨富学 Yang Fuxue, “一件珍贵的回鹘文寺院经济文书” (Yijian zhengui de huihuwen siyuan jingji wenshu) [Tapınak Ekonomisiyle İlgili Kiymetli Bir Uygurca Belge], Xibei minzu yanjiu, 1992, say1 1, s. 59-65.

刘戈 Liu Ge, “回鹘文社会经济文书研究评介” (Huihuwen shehui jingji wenshu yanjiu pingjie) [Uygurca Sosyo-Ekonomik Vesikaların Araştırılması Üzerine Tahlil], Wulumuqi zhiye daxue xuebao, 1992, say1 2, s. 85-90.

刘戈 Liu Ge, “回鹘文社会经济文书研究综述” (Huihuwen shehui jingji wenshu yanjiu zongshu) [Uygurca Sosyo-Ekonomik Vesikalar Üzerinde Araştırmalar], Xiyu yanjiu, 1992, sayı 3, s. 100103.

李经纬 Li Jingwei，“五件敦煌回鹘文遗书译注” (Wujian dunhuang huihuwen yishu yizhu) [Dunhuang'da Bulunmuş Uygurca Beş Vasiyet Belgesinin Tercümesi ve Açıklaması], Xibei minzu yanjiu, 1992, say1 2, s. 31-59.

李经纬 Li Jingwei, “吐鲁番 IB4672 号回鹘文庙柱文书考释” (Tulufan IB4672 hao huihuwen miaozhu wenshu kaoshi) 
[Turfan'daki IB4672 Numaralı Uygurca Tapınak Kazık Yazıtının Tercümesi Üzerine], Xiyu yanjiu, 1992, say1 4, s. 73-80.

袁丁 Yuan Ding, “回鹘文社会经济文书研究注（六）” (Huihuwen shehui jingji wenshu yanjiu zhu (liu)) [Uygurca SoyoEkonomik Vesikalardan Seçmeler- 6], Kashi shifan xuebao, 1992, sayı 1, s. 48-60.

\section{3}

牛汝极 Niu Ruji, 杨富学 Yang Fuxue, “五件回鹘文摩尼教文献 考释” (Wujian huihuwen monijiao wenxian kaoshi) [Mani Diniyle İlgili Beş Uygurca Belgenin Açıklaması Üzerine], Xinjiang daxue xuebao, 1993, say1 4, s. 109-115.

Hiroshi Umemura, Çev: Yang Fuxue, “中华人民共和国藏回鹘文 写本” (Zhonghua renmin gongheguo cang huihuwen xieben) [Çin Halk Cumhuriyeti'nde Muhafaza Edilen Uygurca El Yazma Belgeleri Üzerine], Xibei minzu yanjiu, 1993, say1 2, s. 151-161.

李经纬 Li Jingwei, “敦煌回鹘文遗书五种” (Dunhuang huihuwen yishu wuzhong) [Dunhuang'da Bulunmuş Uygurca Beş Vasiyet Belgesi Üzerine], Xiyu yanjiu, 1993, say1 2, s. 31-45.

齐陈骏 Qi Chenjun, “敦煌，吐鲁番文书中有关法律文化资料简 介” (Dunhuang, tulufan wenshu zhong youguan falü wenhua ziliao jianjie) [Dunhuang, Turfan Vesikalarında Hukuk Kültürüyle İlgili Kaynakların Tanıtımı], Dunhuang xue biankan, 1993, sayı 1, s. 110.

1994

牛汝极 Niu Ruji, “维吾尔学研究的丰碑—“回鹘文契约文书集成” 评介” (Weiwuerxue yanjiu de fengbi — 'huihuwen qiyue wenshu 
jicheng' pingjie" [Uygurca Araştırmaları Alanındaki Önemli Eser —'Uygurca Sözleşme Belgelerinin Koleksiyonu'], Xiyu yanjiu, 1994, say1 4, s. 129-134.

牛汝极 Niu Ruji, 杨富学 Yang Fuxue, “敦煌出土早期回鹘语世 俗文献译释” (Dunhuang chutu zaoqi huihuyu shisu wenxian yishi) [Dunhuang'da Bulunmuş Erken Döneme Ait Uygurca Din Dışı Belgelerinin Tercümesi ve Açıklaması], Dunhuang yanjiu, 1994, say1 4, s. 7-27.

杨富学 Yang Fuxue, “德国新刊布的几件回鹘文租佃契约” (Deguo xinkanbu de jijian huihu wen zudian qiyue) [Almanya'da Yayımlanan Birkaç Uygurca Kira Sözleşmesi Üzerine], Wenshi, 1994, s. $276-285$.

杨富学 Yang Fuxue, “敦煌出土回鹘语浐语” (Dunhuang chutu huihuyu yanyu) [Dunhuang'da Bulunmuş Uygurca Belgelerde Geçen Atasözleri Üzerine], Sheke zongheng, 1994, say1 4, s. 52-54. 李经纬 Li Jingwei, “回鹘文社会经济文书研究” (Huihuwen shehui jingji wenshu yanjiu) [Uygurca Sosyo-Ekonomik Vesikalar Üzerinde Araştırma], Kashi shifan xueyuan xuebao, 1994, sayı 2, s. 36-55.

李经纬 Li Jingwei, “回鹘文社会经济文书研究 (续)” (Huihuwen shehui jingji wenshu yanjiu (xu)" [Uygurca SosyoEkonomik Vesikalar Üzerinde Araştırma 'Devamı'], Kashi shifan sueyuan хиеbao, 1994, say1 4, s. 51-67.

李经纬 Li Jingwei, “回鹘文奴隶买卖与人口典押文书五种” (Huihuwen nuli maimai yu renkou dianya wenshu wuzhong) [Köle 
Alım-Satımı ve Rehinle İlgili Uygurca Beş Vesika], Xibei minzu уапjiu, 1994, say1 2, s. 1-14.

李经纬 Li Jingwei, “四件回鹘文社会经济文书译注” (Sijian huihuwen shehui jingji wenshu yizhu) [Dört Uygurca SosyoEkonomik Vesikanın Tercümesi ve Notlar], Xiуu yanjiu, 1994, say1 2, s. 66-77.

李经纬 Li Jingwei, “回鹘文社会经济文书选注（五）-各类经济 记录” (Huihuwen shehui jingji wenshu xuanzhu (wu)- gelei jingji jilu) [Uygurca Sosyo-Ekonomik Vesikalardan Seçmeler (5)- Çeşitli İktisadi Kayıtlar], Kashi shiyuan xuebao (Zhexue shehui kexueban), 1994, say1 3, s. 69-79.

Nobuo Yamada, Çince çev: 朱悦梅 Zhu Yuemei, “大谷探险队携 归回鹘文买卖借贷契约” (Dagu tanxiandui xiegui huihuwen maimai jiedai qiyue) [Ōtani Kōzui'un Kazı Ekibinden Kalan Uygurca Alım-Satım ve Borç Vesikaları Üzerine], Dunhuang yanjiu, 1994, say1 1, s. 95-208.

齐陈骏 Qi Chenjun, “有关遗产继承的几件敦煌遗书” (Youguan yichan jicheng de jijian dunhuang yishu) [Mal-Mülk Veraseti İle İlgili Dunhuang'da Bulunmuş Birkaç Vasiyet Belgesi], Dunhuangxue biankan, 1994, say1 2, s. 51-60.

\section{5}

李经纬 Li Jingwei, 靳尚怡 Jin Shangyi, “回鹘文文献语言的后置 词” (Huihuwen wenxian yuyan de houzhici) [Uygurca Vesikalarında Son Ekler], Yuyan yu fanyi, 1995, sayı 1, s. 16-22. 李经纬 Li Jingwei, “九件回鹘文社会经济文书译释” (Jiujian huihuwen shehui jingji wenshu yishi) [Dokuz Uygurca Sosyo- 
Ekonomik Vesikasının Tercümesi ve Açılaması], Kashi shifan xueyuan xuebao, 1995, say1 1, s. 32-56.

李经纬 Li Jingwei，“回鹘文借贷文书七种” (Huihuwen jiedai wenshu qizhong) [Borç Alımıyla İlgili Yedi Uygurca Belge], Kashi shifan xueyuan xuebao, 1995, say1 3, s. 30-38.

李经纬 Li Jingwei, “吐鲁番回鹘文买卖文书四种” (Tulufan huihuwen maimai wenshu sizhong) [Turfan Uygur Vesikalarından Alım-Satım İlgili Dört Belge], Xiуu yanjiu, 1995, sayı 2, s. 26-34. 李经纬 Li Jingwei，“回鹘文社会经济文书选注(五)” (Huihuwen shehui jingji wenshu xuanzhu (wu)) [Uygurca Sosyo-Ekonomik Vesikalardan Seçmeler-5], Kashi shifan xueyuan xuebao, 1995, say1 2, s. 43-49.

杨富学 Yang Fuxue, 牛汝极 Niu Ruji，沙州回鹘及其文献 (Shazhou huihu jiqi wenxian) [Shazhou Uygurları ve Onların Eserleri], Gansu: Gansu wenhua chubanshe, 1995.

\section{6}

李经纬 Li Jingwei, “回鹘文社会经济文书的发现, 收藏与研究情 况概述” (Huihuwen shehui jingji wenshu de faxian, shoucang yu yanjiu qingkuang gaishu) [Uygurca Soso-Ekonomik Vesikalarının Bulunma, Muhafaza Edilme ve Araştırılma Durumu Üzerine], Kashi shifan xueyuan xuebao, 1996, sayı 2, s. 66-80.

李经纬 Li Jingwei，吐鲁番回鹘文社会经济文书研究 (Tulufan huihuwen shehui jingji wenshu yanjiu) [Turfan Uygurlarının Sosyo-Ekonomik Vesikaları Üzerinde Araştırma], Ürümçi: Xinjiang daxue chubanshe, 1996. 
1997

牛汝极 Niu Ruji, “国外对维吾尔文献的收藏及研究” (Guowai dui weiwu'er wenxian de shoucang ji yanjiu) [Yurt Dışındaki Uygurca Vesikaların Muhafaza Edilme ve Araştırılma Durumu] Xiуu yanjiu, 1997, say1 2, s. 54- 65.

杨富学 Yang Fuxue, “回鹘文献所见蒙古”合罕”称号之使用范围” (Huihu wenxian suojian menggu 'hehan' chenghao zhi shiyong fanwei) [Uygurca Belgelerde Geçen Moğolca “Kağan” Unvanının Kullanım Alanlar1], Neimenggu shehui kexue, 1997, sayı 5, s. 4446.

郑炳林 Zheng Binglin，杨富学 Yang Fuxe, “敦煌西域出土回鹘 文文献所载 qunbu 与汉文文献所见官布研究” (Dunhuang xiyu chutu huihuwen wenxian suozai qunbu yu hanwen wenxian suojian guanbu yanjiu) [Dunhuang ve Batı Bölgelerinde Bulunmuş Uygurca Vesikalarda Tespit Edilen Qunbu Kelimesi ve Çince Vesikalarda Geçen Guanbu Kelimesi Üzerinde Araştırma], Dunhuangxue jikan, 1997, Say1 2, s. 19-27.

刘戈 Liu Ge,“回鹘文文书的特点和史料价值” (Huihuwen wenshu de tedian he shiliao jiazhi) [Uygurca Vesikaların Özelliği ve Tarihi Değeri], Xiyu yanjiu, 1997, say1 4, s. 120-124.

刘戈 Liu Ge, Aziz Yüsüp, “回鹘文文书研究与汉语译音问题” (Huihuwen wenshu yanjiu yu hanyu yiyin wenti) [Uygurca Belgelerin Araştırılması ve Çince Transkripsiyonu Üzerine], Xiyu yanjiu, 1997, say1 2, s. 75-81.

李经纬 Li Jingwei，“回鹘文社会经济文书的发现、收藏与研究 情况概述” (Huihuwen shehui jingji wenshu de faxian, shoucang 
yu yanjiu qingkuang gaishu) [Uygurca Sosyo-Ekonomik Vesikaların Keşfi, Muhafaza Edilmesi ve Araştırılma Durumuna Genel Bakış], Xibei minzu yanjiu, 1997, sayı 1, s. 157-171.

\section{8}

刘戈 Liu Ge，“从格式与套语看回鹘文买卖文书的年代” (Cong geshi yu taoyu kan huihuwen maimai wenshu de niandai) [Kalıp İfadelerden Uygur Alım-Satım Vesikalarının Tarihine Bakış], Xiyu yanjiu, 1998, say1 2, s. 64-72.

刘戈 Liu Ge, “回鹘文买卖文书纪年月日研究” (Huihuwen maimai wenshu ji nian yue ri yanjiu) [Uygurca Alım-Satım Vesikalarındaki Y1l, Ay ve Gün Üzerinde İnceleme], Minzu yanjiu, say1 5, s. 91-96.

李经纬 Li Jingwei, “莎车出土回鹘文土地买卖文书译释” (Shache chutu huihuwen tudi maimai wenshu yishi) [Yarkend'de Bulunmuş Uygurca Toprak Alım-Satım Belgesinin Tercümesi ve Açıklamas1], Xiyu yanjiu, 1998, say1 3, s. 18-28.

李天石 $\mathrm{Li}$ Tianshi, “敦煌所出卖身、典身契约年代考” (Dunhuang suochu maishen, dianshen qiyue niandai kao) [Dunhuang'da Bulunmuş Satılma ve Rehin Olma Belgesinin Tarihlendirilmesi Üzerine Tahlil], Dunhuangxue biankan, 1998, say1 1, s. 25-30.

杨富学 Yang Fuxue, “宋元时代维吾尔族农奴制度的形成与发展” (Song yuan shidai weiwu'erzu nongnu zhidu de xingcheng yu fazhan) [Song ve Yuan Sülalesi Döneminde Uygurlarda Serf Sisteminin Oluşumu ve Gelişimi], Zhongguo weiwuer lishi wenhua 
yanjiu luncong, Ürümçi: Xinjiang renmin chubanshe, 1998, s. 107-119.

\section{9}

刘戈 Liu Ge, “回鹘文买卖文书的格式套语与断代问题” (Huihuwen maimai wenshu de geshi taoyu yu duandai wenti) [Uygurca Alım-Satım Vesikalarında Geçen Kalıp İfadeler ve $\mathrm{Bu}$ Belgelerin Tarihlendirilmesi Üzerine], Xibei shidi, 1999, sayı 1, s. 42-94.

朱悦梅 Zhu Yuemei, “我国回鹘文社会经济文书研究述要” (Woguo huihuwen shehui jingji wenshu yanjiu shuyao) [Ülkemizdeki (Çin'deki) Uygurca Sosyo-Ekonomik Vesikalarla İlgili Araştırmalara Bakış], Dunhuangxue biankan, 1999, sayı 1, s. 132-135.

İsrapil Yüsüp, “吐鲁番出土回鹘语文书两件” (Tulufan chutu huihuyu wenshu liangjian) [Turfan'da Bulunmuş İki Uygurca Vesika], Yayına Haz. Jie Yaohua "Yar Şehrinin Korunması ve Araştırılması", Xinjiang renmin chubanshe, 1999, s. 353-359.

\section{0}

杨富学 Yang Fuxue, “回鹘文社会经济文书研究百年回顾” (Huihuwen shehui jingji wenshu yanjiu bainian huigu) [Uygurca Sosyo-Ekonomik Vesikaların Araştııılmasının Yüzyıllığına Bakış], Dunhuang yanjiu, 2000, say1 4, s. 169-177.

刘戈 Liu Ge, “从回鹘文契约文书看 $13-14$ 世纪高昌地区的民 族或部落” (Cong huihuwen qiyue wenshu kan 13-14 shiji gaochang diqu de minzu huo buluo) [Uygur Sözleşme Belgeleri Temelinde 13.-14. Yüzyıllarda Koço Bölgesinde Yaşayan 
Milletler ve Kabilelere Bakış], Xibei minzu yanjiu, sayı 2, 2000, s. $47-65$.

刘戈 Liu Ge，回鹘文契约文书初探 (Huihuwen qiyue wenshu chutan) [Uygurca Sözleşme Belgeleri Üzerinde İlk Araştırmalar], Taibei: Wunan chubanshe, 2000.

刘戈 Liu Ge, “回鹘文契约文书研究概况及存在的问题” (Huihuwen qiyue wenshu yanjiu gaikuang ji cunzai de wenti) [Uygurca Anlaşma Belgelerinin Araştırılma Durumu ve Saklanan Meseleler], Міпzи уапjiu, 2000, sayı 2, s. 76-84.

王菲 Wang Fei，“回鹘文摩尼教寺院文书”再考释” ('Huihuwen monijiao siyuan wenshu' zai kaoshi) ['Uygurca Mani Dini Tapınak Belgesi’ Üzerinde Yeniden Açıklama], Ouya xuekan, 2000, sayı 2 , s. $225-242$.

陈永胜 Chen Yongsheng, 敦煌吐鲁番法制文书研究 (Dunhuang tulufan fazhi wenshu yanjiu) [Dunhunag, Turfan Hukuk Belgeleri Üzerinde Araştırma], Gansu: Gansu renmin chubanshe, 2000.

\section{1}

杨富学 Yang Fuxue, “敦煌吐鲁番文献所见吐蕃回鹘之文化关系”

(Dunhuang tulufan wenxian suojian tubo huihu zhi wenhua guanxi) [Dunhuang, Turfan Vesikaları Temelinde Tibet - Uygur Kültürel İlişkisi], Shoudu shifan daxue xuebao, 2001, sayı 1, s. 18-24.

Ablikim Yasin, 吐鲁番回鹘文世俗文书语言结构研究 (Tulufan huihuwen shisu wenshu yuyan jiegou yanjiu) [Turfan'da Bulunmuş Uygurca Din Dışı Belgelerin Dil Yapısı Üzerinde İnceleme], Ürümçi: Xinjiang daxue chubanshe, 2001. 
Ablikim Yasin，王正良 Wang Zhengliang，“吐鲁番回鹘文世俗 文书语言数量研究” (Tulufan huihuwen shisu wenshu yuyuan shuliang yanjiu) [Turfan'da Bulunmuş Uygurca Din Dış1 Belgelerinde Geçen Sayılar Üzerinde Araştırma], Xinjiang daxue xuebao (Zhexue shehui kexue xuebao), 2001, s. 133-136

2002

Geng Shimin, “各国收藏的回鹘文文书概况、语言与翻译 (汉 文)" (Geguo shoucang de huihuwen wenshu gaikuang, yuyan yu fanyi (hanwen)) [Çeşitli Ülkelerde Muhafaza Edilen Uygurca Vesikaların Genel Durumu, Dili ve Tercümesi] (Çince), Yuyan yu fanyi, 2002, say1 1, s. 18-19.

刘戈 Liu Ge, “回鹘文契约中的“bil-”与汉文契约中的“知”现象 考” (Huihuwen qiyue zhong de "bil-" yu hanwen qiyue zhong de “zhi” xianxiang kao) [Uygurca Anlaşma Belgelerinde Geçen 'bil'Kelimesiyle Çince Anlaşma Belgelerinde Geçen 'zhi (bilmek)' Kelimesi Üzerine], Minzu yanjiu, 2002, say1 5, s. 69-76.

Ablikim Yasin, “吐鲁番回鹘文世俗文书动词条件式研究” (Tulufan huihuwen shisu wenshu dongci tiaojianshi yanjiu) [Turfan'da Bulunmuş Uygurca Din Dışı Belgelerindeki Fiilin Şart Kipi Üzerinde Araştırma], Yuyan yu yanjiu, 2002, s. 24-25.

傲奇 Jiao Qi, “研究回鹘文契约文书的创新之作——读“回鹘文 契约文书初”（Yanjiu huihuwen qiyue wenshu de chuangxin zhizuo — du 'huihuwen qiyue wenshu chu) [Uygurca Sözleşme Belgeleri Üzerinde Yapılan Yenilikçi Çalışma—_Uygurca Sözleşme Belgeleri Üzerinde Araştırmalar'], Zhongguo bianjiang shidi yanjiu, 2002, say1 2, s. 102-103. 
劳心 Lao Xin, “从敦煌文献看 9 世纪后的西州——兼论吐鲁番 出土回鹘文木杵文书年代和沙州回鹘的兴衰” (Cong dunhuang wenxian kan 9 shijihou de xizhou—_ jianlun tulufan chutu huihuwen muchu wenshu niandai he shazhou huihu de xingshuai) [Dunhuang Belgeleri Temelinde 9. Yüzyıldan Sonraki Xizhou'a (Batı Aymak) Bakış_ — Turfan'da Bulunmuş Uygurca Kazık Yazıtlarının Tarihlendirilmesi ve Shazhou Uygur Devletinin Yükselişi ve Çöküşü], Dunhuang yanjiu, 2002, sayı 1, s. 81-112.

陈永胜 Chen Yongsheng, “敦煌法制文书研究回顾与展望” (Dunhuang fazhi wenshu yanjiu huigu yu zhanwang) [Dunhuang Hukuk Belgelerinin Araştırılmasına Bakış ve Beklentiler], Dunhuang yanjiu, 2002, say1 2, s. 100-104.

\section{3}

刘戈 Liu Ge, “回鹘文契约上的倒写文字” (Huihuwen qiyue shang de daoxie wenzi) [Uygurca Anlaşma Belgelerindeki Ters Yazılar Üzerine], Minzu yanjiu, 2003, say1 5, s. 51-58.

杨富学 Yang Fuxue, 回鹘文献与回鹘文化 (Huihu wenxian yu huihu wenhua) [Uygurca Vesikalar ve Uygur Kültürü], Pekin: Minzu chubanshe, 2003.

\section{4}

杨富学 Yang Fuxue, “敦煌吐鲁番文献所见回鹘古代历法” (Dunhuang tulufan wenxian suojian huihu gudai lifa) [Dunhuang ve Turfan Vesikalarında Geçen Uygurların Eski Takvimleriyle İlgili Bilgiler Üzerine], Qinghai minzu xиeyuan xuebao, 2004, say1 4, s. 118-123. 
刘戈 Liu Ge, “回鹘文契约证人套语研究” (Huihuwen qiyue zhengren taoyu yanjiu) [Uygurca Anlaşma Vesikalarındaki Şahidin Kullandığı Kalıp İfadeler Üzerinde Araştırma], Minzu yanjiu, 2004, say1 5, s. 55-63.

刘戈 Liu Ge, “从 bitidim (我写的) 看回鹘人的文化” (Cong bitidim (wo xie de) kan huihuren de wenhua) ['Bitidim' Kelimesinden Uygur Kültürüne Bakış], Zhongguo minzu guwenzi yanjiu hui diqici xueshu yantao hui lunwen ji, 2004, s. 1-7.

霍存福 Huo Cunfu, 章燕 Zhang Yan, “吐鲁番回鹘文借贷契约研 究” (Tulufan huihuwen jiedai qiyue yanjiu) [Turfan'da Bulunmuş Uygurca Ödünç Alma Vesikalarının Araştırılması Üzerine], Jilin daxue shehuixue xuebao, 2004, say1 6, s. 95-106.

霍存福 Huo Cunfu, 王宏庆 Wang Hongqing, “吐鲁番回鹘文买卖 契约分析” (Tulufan huihuwen maimai qiyue fenxi) [Turfan Uygurca Alım-Satım Vesikalarının Analizi], Dangdai faxue, 2004, say1 1, s. 5-18.

李树辉 Li Shuhui, “回鹘文摩尼教寺院文书写作年代及相关史 事研究” (Huihuwen monijiao siyuan wenshu xiezuo niandai ji xiangguan shishi yanjiu) [Uygurca Mani Dini Tapınak Belgesinin Yazıldı̆̆ı Dönem ve Onun Tarihiyle İlgili Araştırmalar], Xibei minzu yanjiu, 2004, sayı 3, s. 14-22.

敏春芳 Mei Chunang, “敦煌契约文书中的“证人’, ‘保人’流变考 释” (Dunhuang qiyue wenshu zhong de 'zhengren', 'baoren' liubian kaoshi) [Dunhuang Sözleşme Belgelerindeki Şahit ve Kefillerin Değişimi Üzerinde Araştırma], Dunhuangxue biankan, 2004, say1 2, s. 99-112. 


\section{5}

刘戈 Liu Ge，“回鹘文契约三种文字的译本及其存在的问题” (Huihuwen qiyue sanzhong wenzi de yiben jiqi cunzai de wenti) [Uygurca Sözleşme Belgelerindeki Üç Çeşit Yazının Transkripsiyonu ve Saklanan Meseleler], Ipek Yolundaki Milletlerin Eski Yazısı ve Kültürü Akademik Sempozyumu Makaleler Toplamı, 2005, sayfa 22-30.

刘戈 Liu Ge, “释回鹘文契约中的 y(a)g(a)n” (Shi huihuwen qiyue zhong de y(a)g(a)n) [Uygur Sözleşme Belgelerinde Geçen y(a)g(a)n Üzerine Açıklama], 2005, Minzu Yanjiu, sayı 6, s. 69-72. 刘戈 Liu Ge，“回鹘文契约中的 pao(s)(I)n, tanuq, k(o)rüp oluryu(c)i" (Huihuwen qiyue zhong de pao(s)(I)n, tanuq, k(o)rüp oluryu(c)i) [Uygurca Sözleșme Belgelerindeki pao(s)(I)n, tanuq, k(o)rüp oluryu(c)i Sözcükleri Üzerine], Dunhuangxue biankan, 2005, 4(4), s. 92-96.

刘戈 Liu Ge, “回鹘文契约文书中的“元契”考” (Huihuwen qiyue wenshu zhong de 'yuanqi' kao) [Uygurca Anlaşma Belgelerindeki 'Baş Bitig' Üzerine], Shanxi shifan daxue xuebao, 2005, sayı 5, s. 74-80.

\section{6}

耿世抿 Geng Shimin, 回鹘文社会经济文书研究 (Huihuwen shehui jingji wenshu yanjiu) [Uygurca Sosyo-Ekonomik Vesikalar Üzerinde Araştırma], Pekin: Zhongyang minzu daxue chubanshe, 2006.

刘戈 Liu Ge, “吐鲁番回鹘文买卖契约所展示的重要社会经济文 化状况” (Tulufan huihuwen maimai qiyue suo zhanshi de 
zhongyao shehui jingji wenhua zhuangkuang) [Turfan'da Bulunmuş Uygurca Alım- Satım Vesikalarının Yansıttığı SosyoEkonomik ve Kültürel Durum], Zhongguo jingjishi yanjiu, 2006, say1 4, s. 104-109.

刘戈 Liu Ge, 回鹘文买卖契约译注 (Huihuwen maimai qiyue yizhu) [Uygurca Alım-Satım Vesikalarının Açıklaması], Zhonghua shuju, 2006.

刘戈 Liu Ge, 燕海雄 Yan Haixiong, “古代吐鲁番地区普通妇女 在社会经济生活中的地位一以吐鲁番出土汉文、回鹘文契约 文书所见为例” (Gudai tulufan diqu putong funü zai shehui jingji shenghuo zhong de diwei-yi tulufan chutu hanwen, huihuwen qiyue wenshu suojian wei li) [Kadim Turfan Bölgesindeki Kadınların Sosyal ve İktisadi Yaşam İçerisindeki KonumuTurfan'da Bulunan Çince, Uygurca Sözleşme Belgeleri Temelinde], Minzu yanjiu, 2006, say1 6, s. 53-62.

张铁山 Zhang Tieshan, “耿世民著〈回鹘文社会经济文书研究〉 评介” (Geng Shimin zhu 〈huihuwen shehui jingji wenshu yanjiu〉 pingjie) [Geng Shimin'in 'Uygurca Sosyo-Ekonomik Vesikalar Üzerinde Araştırma' Adlı Kitabı Üzerinde Değerlendirme], Xiyu yanjiu, 2006, say1 4, s. 113-115.

\section{7}

杨富学 Yang Fuxue, “回鹘文文献与高昌回鹘经济史的构建” (Huihuwen wenxian yu gaochang huihu jingjishi de goujian) [Uygurca Vesikalar ve Koço Uygurlarının İktisat Tarihinin Oluşumu], Shixue shi yanjiu, 2007, sayı 4, s. 106-111. 
张铁山 Zhang Tieshan, “刘戈著〈回鹘文买卖契约译注〉评介” (Liu Ge zhu 〈huihuwen maimai qiyue yizhu〉 pingjie) [Liu Ge'in 'Uygurca Alım-Satım Vesikalarının Açıklaması' Adlı Eseri Üzerinde Değerlendirme], Xiyи yanjiu, 2007, sayı 3, s. 124-127.

李志军 Li Zhijun, “高昌回鹘的农业及相关问题研究” (Gaochang huihu de nongye ji xiangguan wenti yanjiu) [Koço Uygurlarında Tarım ve Bununla İlgili Araştırmalar], Xibei minzu daxue shuoshi lunwen, 2007.

2009

李畅 Li Chang, “敦煌吐鲁番出土遗嘱探析” (Dunhuang tulufan chutu yizhu tanxi) [(Dunhuang, Turfan'da Bulunmuş Vasiyet Belgeleri Üzerinde Araştırma], Shanxi xifanxue shuoshi lunwen, 2009.

2010

刘戈 Liu Ge, “回鹘文契约中的(c)(i)n bitig 为汉语“亲契”考” (Huihuwen qiyue zhong de (c)(i)n bitig wei hanyu 'qinqi' kao) [Uygurca Sözleşme Belgelerinde Geçen 'Çın Bitig' Kelimesi ve Çince 'Qin Qi' Kelimesi Üzerine], Minzu yanjiu, 2010, sayı 1, s. $72-83$.

敖特根 Ao Tegen, “莫高窟北区出土回鹘蒙古文卖身契约残片” (Mogaoku beiqu chutu huihu mengguwen maishen qiyue canpian) [Mogao Mağara Külliyesinin Kuzey Bölgesinden Ele Geçirilen Uygurca-Moğolca Satılma Vesikasının Kalıntıları Üzerine], Dunhuang yanjiu, 2010, s. 111-116. 


\section{1}

也小红 Nie Xiaohong, “中古西域民汉文买卖契约比较研究” (Zhonggu xiyu minhanwen maimai qiyue bijiao yanjiu) [Kadim Batı Bölgesindeki Milletlere Ait Alım-Satım Vesikaları ile Çince Alım-Satım Vesikalarının Karşılaştırmalı İncelemesi], Xiyu yanjiu, 2011, say1 2, s. 55-62.

罗海山 Luo Haishan, “回鹘文契约“官罚”内容研究” (Huihuwen qiyue 'guanfa' neirong yanjiu) [Uygurca Sözleşme Belgelerindeki Cezai Uygulamaların İçeriği Üzerinde Araştırma], Guizhou shehui kехие, 2011, say1 9, s. 123- 127.

杨淑红 Yang Shuhong, “元代契约文书的刊布与研究综述” (Yuandai qiyue wenshu de kanbu yu yanjiu zongshu) [Yuan Dönemindeki Sözleşme Belgeleri Üzerinde Yapılan Yayınlar ve Araştırmalar], Zhongguoshi yanjiu dongtai, 2011, sayı 1, s. 28-34.

\section{2}

李经纬 Li Jingwei，回鹘文社会经济文书辑解 (Huihuwen shehui jingji wenshu jijie) [Uygurca Sosyo-Ekonomik Belgelerin Edisyon Kritiği ve Açıklaması], Lanzhou: Gansu minzu chubanshe, 2012. 刘艺铭 Liu Yiming, “吐鲁番出土文书研究综述” (Tulufan chutu wenshu yanjiu zongshu) [Turfan'da Bulunmuş Belgelerin Araştırılma Durumu Üzerine], Yishu yanjiu, 2012, sayı 6, s. 80-82

2013

朱国祥 Zhu Guoxiang, “回鹘文世俗文书中汉语借词对音研究一 以山田信夫和李经纬著版本为例” (Huihuwen shisu wenshu zhong hanyu jieci duiyin yanjiu—yi Shantianxinfu he Li Jingwei zhu banben wei li) [Uygurca Din Dışı Belgelerindeki Çince Alıntı 
Sözcüklerin Ses Bilimsel Açıdan Karşılaştırmalı İncelenmesi Nobuo Yamada ve Li Jingwei'in Yayınları Temelinde], Jiangxi jiaoyu хиеуuan xuebao (Toplam), 2013, say1 6, s.154- 158.

Pezilet Memetcan, “浅析回鹘文买卖契约的法律性质一以“阿狄 赫达干卖地契, 为例” (Qianxi huihuwen maimai qiyue de falü xingzhi-yi 'adihedagan maidiqi' wei li) [Uygurca Alım-Satım Belgelerinin Yasal Karakteri Üzerinde Tahlil—_Adihedagan'in Tarla Satış Sözleşmesi Örneğiyle'], Hetian shikan zhuke xuexiao xuebao, 2013, say1 4, s.112-114.

\section{4}

付马 Fu Ma, “回鹘时代的北庭城一德藏 Mainz 354 号文书所见 北庭城重建年代考” (Huihu shidai de beitingcheng - de cang Mainz 354 hao wenshu suojian beiting cheng chongjian niandai kao) [Uygur Döneminde Beşbalık Şehri-Almanya'da Muhafaza Edilen Mainz 354 No'lu Belgedeki Bilgiler Temelinde Beşbalık Şehrinin Restorasyon Tarihi Üzerine Araştırma], Xiyu yanjiu, 2014, say1 2, s. 9-22.

\section{5}

于佳音 Yu Jiayin, “回鹘文社会经济文书 tamga 和 nişan 研究” (Huihuwen shehui jingji wenshu tamga he nişan yanjiu) [Uygurca Sosyo-Ekonomik Vesikalarda Geçen Tamga ve Nişan Üzerinde Araştırma], Zhongyang minzu daxue, shuoshi lunwen, 2015.

\section{6}

也小红 Nie Xiaohong, “试论回鹘文契约的前后期之分” (Shilun huihuwen qiyue de qianhou qizhi fen) [Uygurca Anlaşma 
Belgelerinin Tarihlendirilmesi Üzerine], Xiyu yanjiu, 2016, sayı 3, s. 24-29.

李阳 Li Yang, “回鹘文献所见高昌回鹘的植棉与绵织” (Huihu wenxian suojian gaochang huihu de zhimian yu mianzhi) [Uygurca Vesikalar Işığında Koço Uygurlarında Pamuk Yetiştiriciliği ve Bez Dokumac1lığ1], Xibei minzu daxue shuoshi xuewei lunwen, 2016.

Ablacan Himit, “回鹘文契约文书量词研究” (Huihuwen qiyue wenshu liangci yanjiu) [Uygurca Vesikalarda Geçen Ölçü Birimleri Üzerine Araştırma], Zhongyang minzu daxue, shuoshi xuewei lunwen, 2016.

\section{7}

张铁山 Zhang Tieshan, 崔炎 Cui Yan “回鹘文契约文书参与者称 谓考释——兼与敦煌吐鲁番汉文文书比较” (Huihuwen qiyue wenshu canyuzhe chengwei kaoshi_ jian yu dunhuang tulufan hanwen wenshu bijiao) [Uygurca Anlaşma Belgelerinde Anlaşmaya Dâhil Olan Taraflar ve Onların Adları. -Dunhuang, Turfan'da Bulunmuş Çince Belgelerle Karşılaştırmalı Olarak], Xiyu yanjiu, 2017, say1 2, s. 79-84.

Mubarek İskender, “回鹘文文献中的对偶词——兼与现代维吾 尔语比较” (Huihuwen wenxianzhong de dui'ouci- jian yu xiandai weiwueryu bijiao) [Uygurca Vesikalarda Geçen Z1t Anlamlı Kelimeler Üzerine —Modern Uygurca ile Karşılaştırmalı Olarak], Zhongyang minzu daxue, shuoshi lunwen, 2017. 
2018

李阳 Li Yang, 杨富学 Yang Fuxe, “高昌回鹘植棉业及其在世 界棉织史上的地位” (Gaochang huihu zhimianye jiqi zai shijie mianzhishi shang de diwei) [Koço Uygurlarının Pamuk Yetiştiriciliği ve Onun Dünya Dokumacılık Tarihindeki Yeri], Shihezi daxue xuebao, 2018, say1 1, s. 95-105.

杨富学 Yang Fuxe, 单超成 Shan Chaocheng, “高昌回鹘王国棉织 业考析” (Gaochang huihu wangguo mianzhiye kaoxi) [Koço Uygur Devletinde Bez Dokumacılığı Üzerine Tahlil], Tulufanxue yanjiu, 2018, say1 1, s. 41-49

单超成 Shan Chaocheng, “高昌回鹘王国的手工业发展研究” (Gaochang huihu wangguo de shougongye fazhan yanjiu) [Koço Uygur Devletinde El Sanatlarının Gelişimi Üzerine Araştırma], Xibei minzu daxue shuoshi xuewei lunwen, 2018.

2019

韩树伟 Han Shuwei, “吐鲁番, 敦煌出土回鹘文契约文书研究述” (Tulufan, dunhuang chutu huihuwen qiyue wenshu yanjiu shu) [Turfan, Dunhuang'da Bulunmuş Uygurca Belgelerin Araştırılması Üzerine], Xibei minzu luncong, 2019, s. 46-54.

2020

韩树伟 Han Shuwei, “西北出土契约文书所见习惯法比较研究” (Xibei chutu qiyue wenshu suojian xiguan fa bijiao yanjiu) [Bat1 Bölgelerinde Bulunmuş Sözleşme Belgelerinde Geçen Kalıp İfadeler Üzerinde Karşılaştırmalı İnceleme], Lanzhou daxue boshi xuewei lunwen, 2020. 
罗将 Luo Jiang “二十年来敦煌契约文书研究述评与展望 (2000-2020)" (Er'shi nianlai dunhuang qiyue wenshu yanjiu shuping yu zhanwang) [Son 20 Y1l İçerisinde Dunhuang'da Bulunmuş Belgelerin Araştırılma Durumu Üzerine Analiz ve Beklentiler (2000-2020)], Hexi хиеуиапbаo, 2020, say1 4, s. 53-59. 2021

亚黎 Ya Li, “回鹘文契约与回鹘式蒙古文契约文书比较研究” (Huihuwen qiyue yu huihushi mengguwen qiyue wenshu bijiao yanjiu) [Uygurca Anlaşma Belgeleri ile Uygur Yazısıyla Yazılmış Moğolca Anlaşma Belgeleri Üzerinde Karşılaştırmalı İnceleme], Xibei minzu daxue, shuoshi lunwen, 2021.

Sonuç: Görüldüğü gibi, Uygur sivil belgeleri üzerine Çin'de ilk yayın 1954 yilında yapılmış ve 1980'den sonra artarak devam etmiştir. Tespit edebildiğimiz kadarıyla, 1954 ve 2021 yılları arasında Çin'de Uygur sivil belgeleri üzerine toplam 142 Çince çalışma yapılmıştır. Bunlar arasında yayımlanan kitap sayısı 8, yüksek lisans ve doktora tezi sayısı 8 ve çeşitli dergilerde yayımlanan araştırma makalelerinin sayısı ise 124 'dır. $\mathrm{Bu}$ çalışmalar arasında metin okumalarına dayanan söz varlığ çalışması ve bu söz varlığının mevcut dönemdeki tarihi, arkeolojik bilgilerle desteklenmesiyle yapılan disiplinlerarası çalışmalar da bulunmaktadır. 


\section{Kaynakça}

Arat, Reşit Rahmeti (1964), “Eski Türk Hukuk Vesikaları”, Türk Kültürü Araştırmaları, I-1, Ankara, s.5-53.

Ayazlı, Özlem (2016), Eski Uygurca Din Dışı Metinlerin Karşılaştırmalı Söz Varlığı. Ankara: Türk Dil Kurumu.

Clark, Larry V.,(1975), Introduction to the Uyghur Civil Documents of East Turkestan (13th-14thcc.). Ph.D Dissertation, Indiana University (Bloomington).

Geng Shimin (2006). 回鹘文社会经济文书研 (Huihuwen shehui jingji wenshu yanjiu] [Uygurca Sosyo- Ekonomik Vesikalar Üzerinde Araştırmalar], Pekin: Zhongyang minzu daxue chubanshe.

İzgi, Özkan (1987). Uygurların Siyasi ve Kültürel Tarihi (Hukuk Vesikalarına Göre), Ankara: Türk Kültürünü Araştırma Enstitüsü Yayınları.

İzgi, Özkan (2014). Orta Asya Türk Tarihi Araştırmaları, Yayına hazırlayanlar: Erkin Ekrem-Serhat Küçük. Ankara: Türk Tarih Kurumu.

Li Jingwei (1997). 吐鲁番回鹘文社会经济文书研究 (Tulufan huihuwen shehui jingji wenshu yanjiu) [Turfan Uygurlarının Sosyo-Ekonomik Vesikaları Üzerinde Araştırma], Ürümçi: Xinjiang renmin chubanshe.

Özyetgin, A. Melek (2015). İslam Öncesi Uygurlarda Toprak Hukuku, İstanbul: Ötüken Neşriyat.

Raschman, Simone-Christiane (2007). Alttürkische Handschriften. Teil 13: Dokumente, Teil 1. (VOHD, XIII-21), Stuttgart: Franz.

Sayit, Muhemmetrehim, İsrapil Yüsüp (2000). Qedimqi Uyg்ur Yéziqidiki Vesiqiler [Eski Uygur Yazılı Vesikalar], Ürümçi: Şincay Helq Neşriyatı. 